\title{
A method to improve the annotation mechanism based on Readium
}

\author{
Mao Chen ${ }^{1, a}$, Hang Luo ${ }^{2, b}$, Tengbaao He $\mathrm{He}^{3, \mathrm{c}}$, Sanya Liu ${ }^{4, \mathrm{~d}}$ \\ ${ }^{1}$ National Engineering Research Center for E-Learning, Central China Normal University, Wuhan \\ 430079, China \\ ${ }^{2}$ National Engineering Research Center for E-Learning, Central China Normal University, Wuhan \\ 430079, China \\ ${ }^{3}$ National Engineering Research Center for E-Learning, Central China Normal University, Wuhan \\ 430079, China \\ ${ }^{4}$ National Engineering Research Center for E-Learning, Central China Normal University, Wuhan \\ 430079, China \\ aemail:chenmao@mail.ccnu.edu.cn,,email:luohanghs@foxmail.com, \\ cemail:423105172@qq.com, ${ }^{d}$ email: Isy5918@mail.ccnu.edu.cn
}

Keywords: EPUB; Readium; Rangy; Annotation

\begin{abstract}
With the rapid development of information technology, great changes have taken place in people's reading habits, and paper books are being replaced by eBooks. Digital annotation tool allows readers to highlight or insert notes into eBooks during the process of reading, which is an important way for readers to interact with book contents, and can significantly improve reading experience. Readium is an open-source framework with a series of high-performance techniques for EPUB parsing, but the annotation mechanism of it performs poorly. In order to improve the annotation function of Readium, this paper presents a new annotation mechanism based on Rangy library. After the application of proposed method, the problem that annotation can't adjust its position to adapt to the changes of display attributes of an eBook has been successfully solved. Moreover, this paper provides convenient tools for user to customize the annotation effect. All the work in this paper will make the Readium framework more perfect.
\end{abstract}

\section{Introduction}

The idea of eBooks emerged ever since the creation of computer, and the progress of modern computer and network technologies in recent years accelerated the popularity of eBooks[1]. In 2010, sales of eBook readers increased to 300 million in America [2]. In China, outputs of eBooks had kept a great growth speed which was at least 25\% from 2007 to 2011[3]. At present, Amazon sold more eBooks than paper books [4]. E-reading has become an important part of modern lives. In education, eBook has evolved to e-textbook, based on which, electronic schoolbag has been widely applied in class [5].

During traditional reading process, reader can emphasize some important contents by using underscores, or writing marginal notes on the page. Annotation helps readers reach a deeper level of engagement and promotes active reading, which embodies the reader's "dialogue with the text" and makes the reader's thoughts into visible record [6]. In a research about the application of eBooks in class, annotation is regarded as one of the most important features that can promote the process of learning [7, 8]. Annotation has been given new possibilities in information environment. For example, Kindle readers can upload the annotations content to social Medias, which can satisfy reader's socialized reading needs [9]. According to a research, anchored text and annotation-based discussion environments can help deepen discussions of the corresponding text and further reader's understanding of it [10]. Therefore, providing eBook readers with effective and convenient annotation tools can help promote reading experience.

Readium is an open source framework to promote the application of EPUB standard, with SDK and APIs to produce a high performance EPUB reading system [11]. Annotation mechanism has 
been implemented in the framework, but its functions are rather weak and seriously affected user experience, typical features of which is that the annotation on the eBook page cannot adjust itself with the change of display attributes of corresponding text. In order to provide readers with a highly personalized and convenient annotating method with perfect user experience, this paper is trying to improve the annotation mechanism of Readium Based on Rangy library.

\section{Analysis}

\section{Annotation mechanism of Readium.}

Essentially, EPUB is a package of web pages that follows the norms of EPUB. The key to parse an EPUB eBook is to access and display the resources in each page according to the EPUB standards, and the basic process of it is shown in Fig. 1. Resources in EPUB include video, photo, audio, web pages, CSS, JavaScript file and other media files. When user chooses an eBook to open from file system, all the resources in the EPUB will be extracted into system memory by Radium, then the program begins to access information from EPUB norm files, including catalogue, metadata, relative paths of resources etc. After that, the browser engine will send a request to local server asking for EPUB resources, and the local server will get resource from memory and send it to browser engine in the form of file streams. At last, the browser engine will organize all the resources and render a HTML page on display terminal.

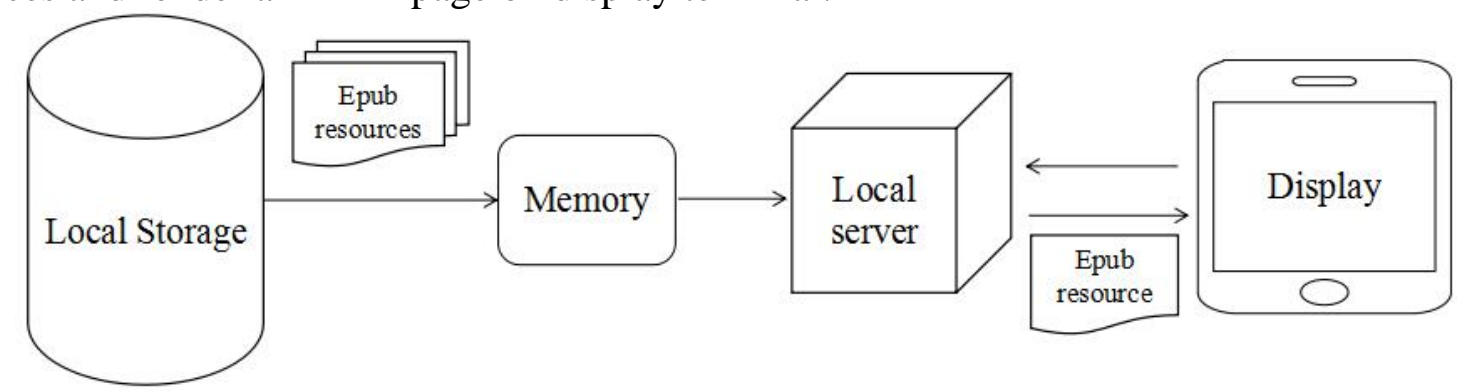

Fig.1.The basic process to display an EPUB of Readium

The annotation function is built on the mechanism of displaying EPUB pages, which is implemented by analyzing the pages in the browser engine and modifying the elements of it. Fig.2 displays the essential steps of annotation mechanism.

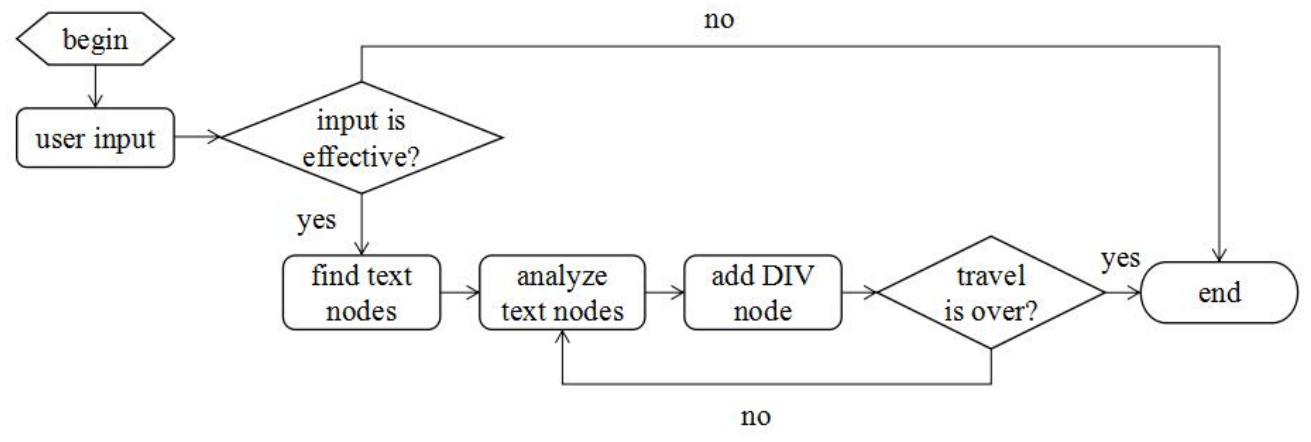

Fig.2.Essential steps of annotation mechanism

Implementation of main steps:

Step 1. User selects the content to be annotated. The way to select book content is different in different platforms. On mobile terminals, such as Pad or smart phone, the user needs to long-press the screen and drag election bar to select the content. In contrast, the user uses mouse to select texts in personal computer. The program will create a range object to store and manipulate user selection and judge whether the range object contains texts [12]. If it contains texts, going to step 2, otherwise the program is over;

Step 2. Go through all the nodes in the range object, and find out all text nodes and put them into an array. Then go through this array and process each text node in this array according to step 3. 
Step 3. Create a range object for each current text node, then call API getClientRects by this range object, which will result in an array of TextRectangle object [13]. Each TextRectangle object is a rectangle model for a line of text, which contains important attributes of this line including height, width and position relative to the document page.

Step 4. Go through the TextRectangle object array, and build a div node according to the data of each TextRectangle object. Thus a div node is created with the same size and position as the text rectangle, and then it is put into the right position with absolute position. The newly added div node will change its style according to cascading style sheet to form various annotations. Fig.3 and Fig. 4 display the highlight annotations effect and underline annotation effect, respectively.

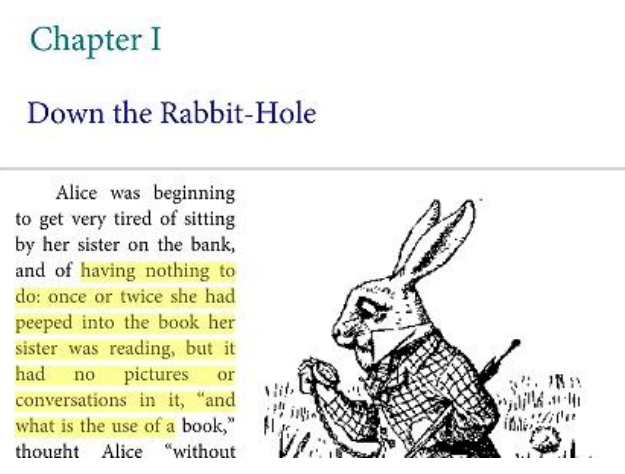

Fig.3.Highlight annotation
Chapter I

\section{Down the Rabbit-Hole}

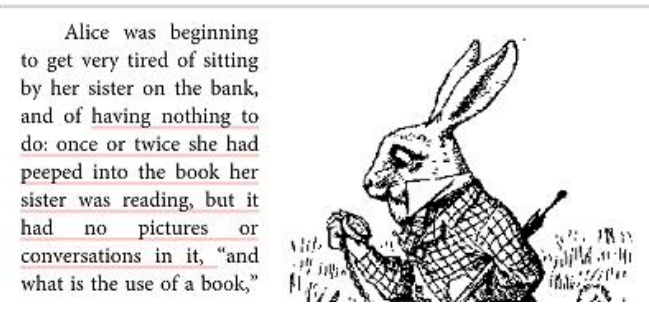

Fig.4.Underline annotation

\section{Existing problems of the annotation mechanism of Radium}

Step 3 and step 4 are essential steps of annotation process in Radium. Annotation attributes can be obtained in step 3, and annotation view is generated in step 4. According to mechanism of Radium, annotation is located with absolute positioning, therefore once an annotation inserts in a page, its position will not change no matter how the attributes of the EPUB page changes, which will lead to the problem of dislocation of annotations. Fig. 5 and Fig. 6 demonstrate two examples that the annotation could not adapt to the changes of font size and page column, respectively.
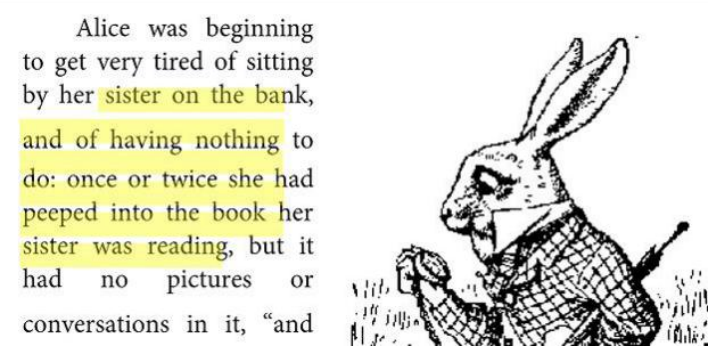

Down the Rabbit-Hole

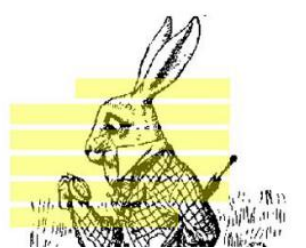

it had no pictures or conversation in it, "and what is the use of a book," thought Alice "without pictures or conversation?"

So she was considering in her own mind (as well as she could, for the hot day made her feel very sleepy and stupid), whether the pleasure of making a daisy-chain would be worth the trouble of getting up an picking the daisies, when suddenly a
White Rabbit with pink eyes ran close by her.

Fig.5. Highlight dislocation after fontsize change Fig.6. Highlight dislocation after column change

\section{Improvement to annotation mechanism}

\section{Method.}

In order to solve above problem, the method of covering a node on texts to simulate the effects of annotation must be changed. A new mechanism that insert span node into book contents to get annotation effects, i.e., insert annotations into texts as a part of the content, is proposed in this paper. Specifically speaking, for each text node in selection area, the program will find out its parent node and then insert a span node into its parent node. Then the program makes span node a container of the text node and changes the view of the text according to the settings of style sheet. The reason to choose span node lies in that it is an in-line element that will not take up a line alone and can stay together with text contents in one line. This mechanism enables that span node to be a part of book contents, effectively eliminating the original influence that the newly added node on the book layout.

\section{Implementation.}

According the idea and mechanism above, the basic process of the improved annotation method 
is shown in Fig. 7, which is based on Rangy library.

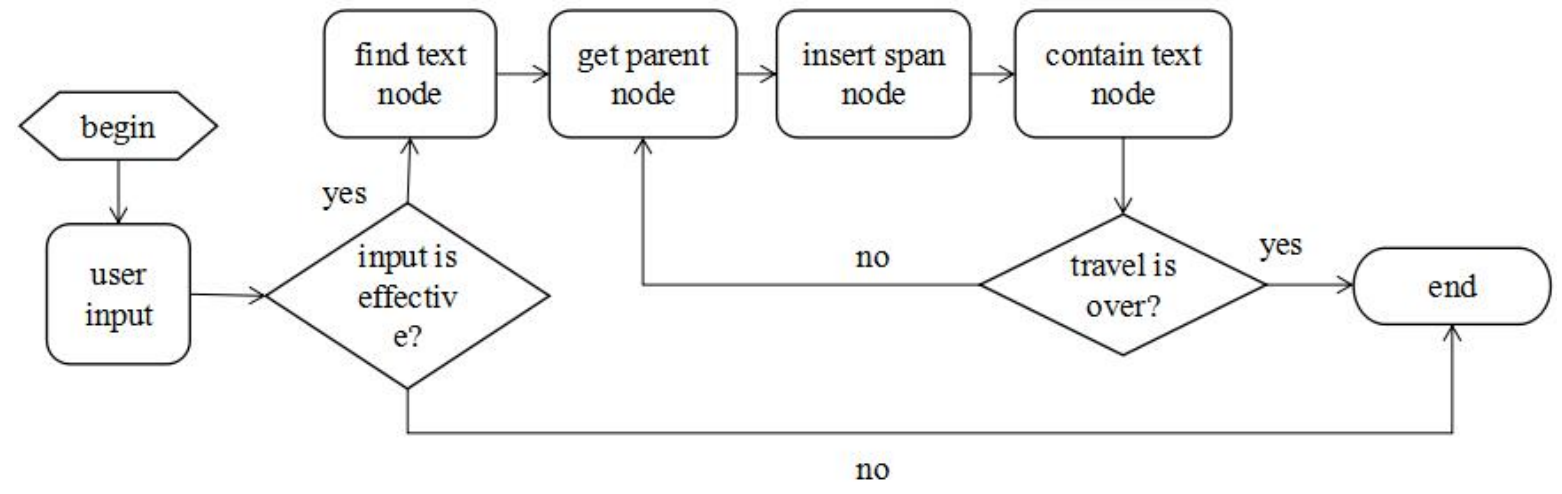

Fig.7. Basic process of improved mechanism

Step 1 and Step2: They are as same as the step1 and step2 in above section;

Step 3. Get parent node by calling the parentNode attribute of the current text node, and get its parent node;

Step 4. Create a span node and insert it into the parent node got at step 3 through API insertBefore, which can be inserted into the parent node with a span node before the current text node;

Step 5. Call API appendChild to insert the current text node into the span node got at step 4. This step will make text node a child node of span. At last, span node changes its inner texts style according to cascading style sheet to form final annotation effects.

Compared with the original mechanism, this method is easier to implement and more convenient. Additionally, it needs less counting steps which may cause some errors.

\section{Personalize annotation}

Annotating is a way of individualized expression, so single and constant annotation style can't meet all the needs of different users. Therefore, personalized annotation are considered and realized in this paper. The method to achieve annotation effects in the proposed mechanism is to insert a cascading style sheet into the document while loading EPUB page. Since cascading styles in the sheet can be covered by adding new styles into the document, modifying the styles of annotation can be realized by adding new cascading style sheet on the EPUB page. Based on this mechanism, the function of setting and modifying attributes of annotation style by user according to personal preference is realized in this paper, and several built-in popular annotation styles are provided for users to choose. Fig. 8 demonstrates the comparison of annotation styles before and after modification.

Alice was beginning to get very tired of sitting by her sister on the bank, and of having nothing to do: once or twice she had peeped into the book her sister was reading, but it had no pictures or conversations in it, "and what is

(a) Annotation before modification
Alice was beginning to get

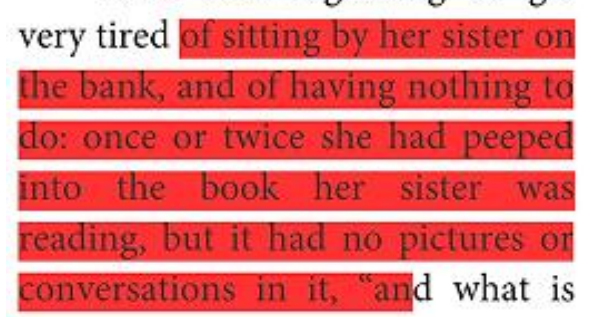

(b) Annotation style after modification

Fig.8. Comparison of two annotation styles

\section{Results}

In order to verify the effectiveness of the new mechanism proposed in this paper, we change the display style of an eBook and check whether the annotation will change automatically to adapt to the corresponding text. 
Fig. 9 displays the original annotations by the proposed mechanism. After increasing the font size and changing the page column from one to two respectively, the annotations change accordingly and match with the text perfectly, as shown in Fig.10 and Fig. 11. Obviously, compared with the dislocated annotation effects in Fig. 5 and Fig. 6, the proposed mechanism can obtain perfect annotation effect, and successfully solve the problem in Radium.

Moreover, as pointed out in above section, we can also get better and personalized annotations by providing user with personalized attribute setting functions.

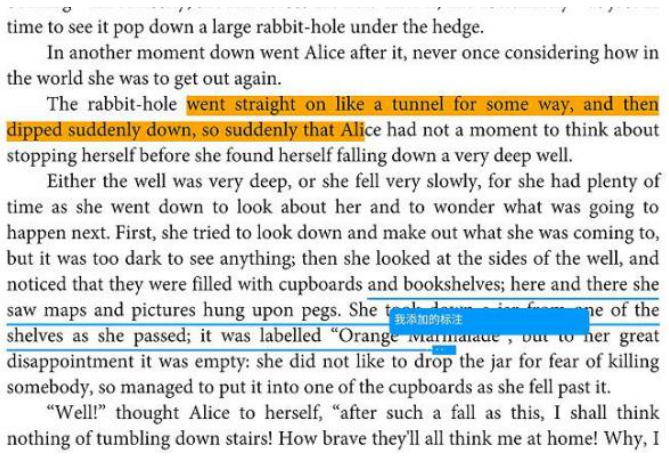

Fig.9 Annotations in a normal eBook

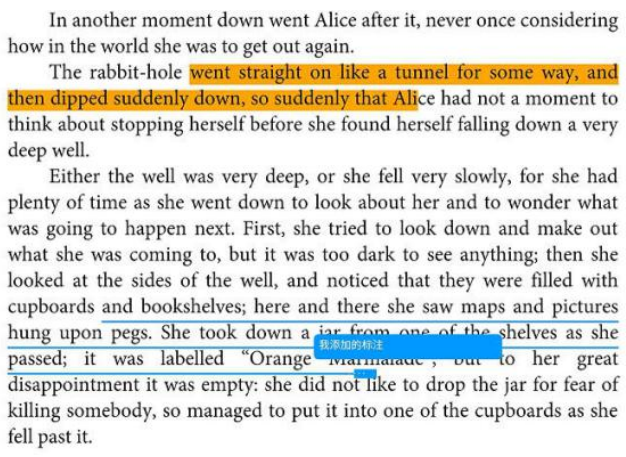

Fig.10. Change fontsize

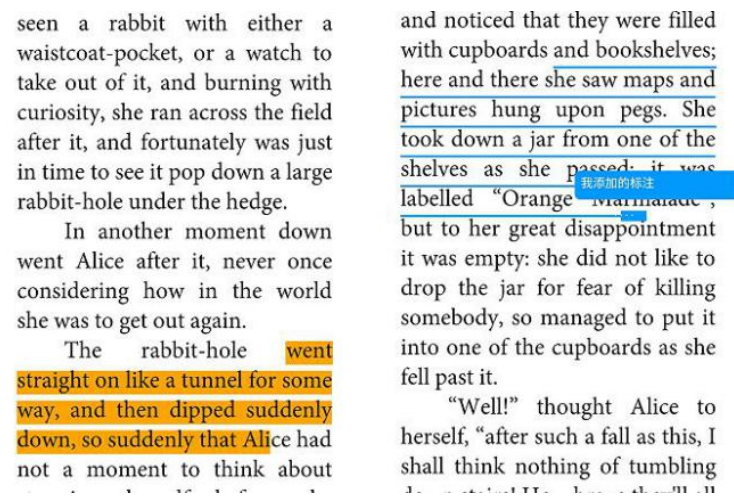

Fig.11. Change display format

\section{Conclusion}

The defect and the mechanism of the annotation in Radium open source framework are analyzed in this paper. A novel method is presented to insert annotations into text contents to make annotations a part of book contents based on Rangy library. Through the comparison with annotation effects in Radium, the results show that the proposed mechanism and method can provide users with better annotation effects and personalizing annotation functions, which can promote reading experiences and meet individual needs. In future works, we hope to add more annotation tools into Readium framework and promote the diversity of annotation styles for users.

\section{Acknowledgement}

The research was sponsored by the National Key Technology Research and Development Program (NO.2014BAH22F01).

\section{References}

[1] Siriginidi Subba Rao. Electronic books: a review and evaluation[J]. Library Hi Tech, 2003, 21(1):85-93.

[2] W.B.Kuang,H.Z.Gong,J.Pu. The development and influence fo Amazon Kindle[J]. Library Theory And Practice, 2011, 5(2): 90-92. 
[3] J.J.Li,Z.Q.Zhang. Situation of the development of eBooks and Trouble analysis[J]. Science And Publishment, 2013, 2(8): 4-9.

[4] David Bounie, Bora Eang,Marvin Sirbu, et al. Superstars and Outsiders in Online Markets: An Empirical Analysis of Electronic Books[J]. Social Science Electronic Publishing, 2012, 12(1):52-59.

[5] Y.H.Wu,Z.T.Zhu,C.He.A Research For Standard Sechnical System Framework Of EBooks And E-bag[N]. Journal of East China Normal University, 2012,3(2).

[6] Carol Porter-O'Donnell. Beyond the Yellow Highlighter: Teaching Annotation Skills to Improve Reading Comprehension[J]. English Journal, 2004, 93(5):8.

[7] Rostislav Fojtik. EBooks and Mobile Devices in Education[J]. Procedia - Social and Behavioral Sciences, 2015, 182:742-745.

[8] Lotta C.Larson. Digital Readers: The Next Chapter in EBook Reading and Response[J]. Reading Teacher, 2010, 64(1):15-22.

[9] D.S.Wang. A research of the promotion of eBooks user experience[J]. News Dissemination, 2015, 6(11): 87-88.

[10] C.M.Chen,F.Y.Chen. Enhancing digital reading performance with a collaborative reading annotation system[J]. Computers \& Education, 2014, 77:67-81.

[11] Readium. Readium Project Goals[EB/OL]. http://readium.org/about/project-goals.

[12] Github. Rangy range[EB/OL]. https://github.com/timdown/rangy/wiki/Rangy-Range.

[13] WEB front-end development. getClientRects returns a TextRectangle object [EB/OL]. http://www.css88.com/archives/4187. 